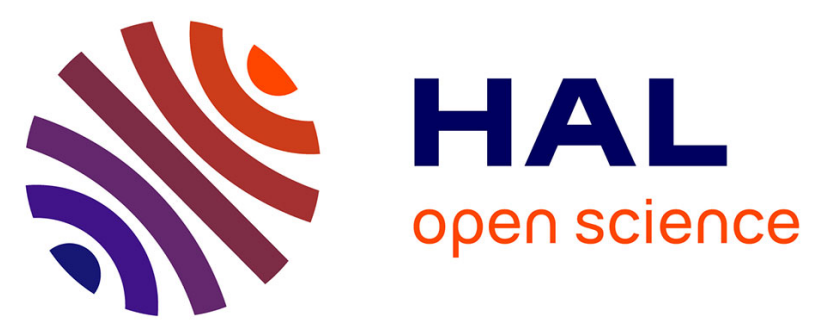

\title{
Assessing the proper color of translucent materials by an extended two-flux model from measurements based on an integrating sphere
}

\author{
Lionel Simonot, Mathieu Hébert, Serge Mazauric, Roger D. Hersch
}

\section{- To cite this version:}

Lionel Simonot, Mathieu Hébert, Serge Mazauric, Roger D. Hersch. Assessing the proper color of translucent materials by an extended two-flux model from measurements based on an integrating sphere. IS\&T International Symposium on Electronic Imaging (EI 2017), Jan 2017, Burlingame, CA, United States. pp.48 - 56, 10.2352/ISSN.2470-1173.2017.8.MAAP-291 . hal-01458760

\author{
HAL Id: hal-01458760 \\ https://hal.science/hal-01458760
}

Submitted on 6 Feb 2017

HAL is a multi-disciplinary open access archive for the deposit and dissemination of scientific research documents, whether they are published or not. The documents may come from teaching and research institutions in France or abroad, or from public or private research centers.
L'archive ouverte pluridisciplinaire HAL, est destinée au dépôt et à la diffusion de documents scientifiques de niveau recherche, publiés ou non, émanant des établissements d'enseignement et de recherche français ou étrangers, des laboratoires publics ou privés. 


\title{
Assessing the proper color of translucent materials by an extended two-flux model from measurements based on an integrating sphere
}

\author{
L. Simonot; Université de Poitiers, Institut Prime UPR CNRS 3346; Futuroscope Chasseneuil, France \\ M. Hébert; Université de Lyon, Université Jean Monnet de Saint Etienne, Laboratoire Hubert Curien UMR CNRS 5516; Saint-étienne, \\ France. \\ S. Mazauric; Université de Lyon, Université Jean Monnet de Saint Etienne, Laboratoire Hubert Curien UMR CNRS 5516, Saint- \\ étienne, France \& CPE Lyon; Villeurbanne, France. \\ R. D. Hersch; École Polytechnique Fédérale de Lausanne (EPFL), Faculté Informatique et Communication, Lausanne, Switzerland
}

\begin{abstract}
The optical and visual characterization of translucent materials according to their shape and thickness is still a challenge for scientific research due to the difficulty to describe and predict in a simple manner the angular and spectral distributions of the reflected and transmitted light. In this paper, the light reflection and transmission properties of slightly scattering polymer sheets stacked with each other are studied. The light that is regularly reflected and transmitted by the material, propagated without scattering, as well as the light which is scattered out of the regular directions, are predicted using only a few measurements from a commercial reflectance/transmittance spectrophotometer and an extended twoflux model of the radiative transfer. The proposed methodology, validated by experiments, can be applied by laboratories equipped with an integrating sphere device which is usually used in the context of strongly diffusing rather than translucent materials. A discussion states the potential and the limitations of the proposed methodology.
\end{abstract}

\section{Introduction}

The color prediction of layered absorbing materials such as those produced by 3D printers is enabled by spectral flux transfer models, among which the widely used two-flux models. However, it is known that the two-flux theory is valid for either nonscattering or strongly scattering media, but not for intermediate levels of scattering. The present contribution aims at extending the classical two-flux model in order to predict the spectral properties, therefore the color, of stacked translucent layers by performing measurements on individual layers with a commercially available integrating sphere reflectance-transmittance spectrophotometer for a better convenience in practical applications.

The optical description of a translucent object is made difficult by the scattering of light occurring in the material itself, and possibly at its interfaces with air. A full characterization requires measuring the angular reflectance and transmittance over the sphere, by distinguishing whenever possible the regularly reflected/transmitted fluxes and the diffuse fluxes. The translucent materials can be classified at the center of the range between non-scattering and strongly scattering materials that are easy to characterize optically.

A) Nonscattering materials with flat interfaces

The propagation of collimated light within a stack of these materials is described by using Beer-Lambert-Bouguer's law for the transmittance within the medium, and Snell laws and Fresnel formulae for the reflectance and transmittance at the interfaces. The material is characterized by its absorption coefficient and its refractive index. A two-flux model, such as the one proposed by Stokes predicting reflectances and transmittances of piles of glass plates. [1], models the multiple reflections of light by the interfaces in the regular directions.

\section{B) Strongly scattering materials}

The propagation of a diffuse (Lambertian) light within strongly scattering materials, characterized by their absorption and backscattering coefficients, can also be modeled by a two-flux model such as the one proposed by Kubelka and Munk [2], or by Kubelka $[3,4]$ in the case of stacked layers.

In the case of translucent materials, the angular distribution of light follows a more complex function and varies as the light propagates through the materials. Light scattering in each point is described by a so-called phase function, incorporated into a multiple scattering model based for example on a resolution of the radiative transfer equation [5,6]. The four-flux method [6-11] is an interesting compromise to solve this equation. It consists in simplifying the light propagation problem by considering four global light components: two components diffused over the upper and lower hemispheres, and two components propagating in the regular reflection and transmission directions in respect to the incidence direction. The proper color of the material is perceived from the diffuse component reflected (respectively transmitted) in the upper (respectively lower) hemisphere. The sensation of gloss (respectively transparency) is perceived from the regularly reflected (respectively transmitted) light component.

In terms of measurement systems, a full optical characterization of a translucent material requires bi-directional measurements over the sphere of the reflectance and transmittance of a slice of material, by using a gonio-spectrophotometer. However, this kind of instrument is expensive and not always available in the industry where one of the main issues is the characterization of the intrinsic color of materials. More classical measurement configurations, recommended by the CIE [12] for color measurements of diffusing materials in reflectance mode, are the $45^{\circ}: 0^{\circ}$ and $0^{\circ}: 45^{\circ}$ geometries where the specimen is illuminated at the first angle and viewed at the second. However, these geometries do not allow relevant color measurement for non-scattering or weakly scattering materials. Only geometries using an integrating sphere allow measuring the regular and diffuse, reflected and transmitted light components. Most sphere based instruments provide the corresponding measurement geometries. However, these configurations require more than a single integrated sphere in order to apply the four-flux method. We present in this paper an 
extended two-flux model (or a degraded four-flux model) which considers the propagation of both diffuse and collimated light fluxes in the material, enables predicting with a rather good accuracy the light components diffused by stacks of translucent layers or sheets, and thereby their intrinsic color. This original method thus overcomes the usual limitations of the two-flux approach by considering intermediate materials between non-scattering and strongly scattering media. The predicted reflectances and transmittances can be directly compared with measurements from an integrating sphere reflectance/transmittance spectrophotometer.

Integrating spheres are spherical cavities coated with a white diffuse coating [13], and are used to measure diffuse reflectance or transmittance of materials. They can be used in two configurations: either in order to illuminate the sample with perfectly diffused light, or in order to collect the light emerging from the sample, thus giving an average value over all angles of illumination or observation. In the scope of the Kubelka-Munk theory, an integrating sphere is often used to produce the needed Lambertian irradiance, whereas the exitance of the scattering material, assumed to be Lambertian, is estimated by measuring only the radiance in one direction (assuming that a same radiance would be measured in every other direction). However, these properties are valid for strongly scattering media but not for translucent, slightly scattering media whose exiting flux is not Lambertian. Reflectance or transmittance predictions based on integrating sphere measurements are therefore not well adapted to translucent materials, unless a specific methodology, such as the one that we introduce in this paper, is applied.

We consider an integrating sphere used for illumination. The integrating sphere has often a black trap located in the symmetric direction to the detector in respect to the normal of the sample. When the trap is open, no light can be specularly reflected from the sample toward the detector: it is the specular component excluded mode $(S C E)$. When the trap is closed, the specularly reflected light is included into the measured radiance: it is the specular component included mode $(S C I)$. The specular excluded reflectance measurement requires the use of the gloss trap, for which the CIE [14] recommends selecting at least all rays within $1^{\circ}$ of the specular reflection direction. Unfortunately, standardization does not exist to more precisely define the angular width of the specular ports. Their angle may vary somewhat depending on the integrating sphere device. The transmittances can also be measured with a diffuse illumination supplied by an integrating sphere. According to the position of the sample close to either the sphere or the detector, the total, respectively the regular, transmittance is obtained. For the regular transmittance, the CIE recommends that the irradiating and measuring geometry be identical with circular cones having a halfangle of $5^{\circ}[14]$.

Using measurements from an integrating sphere, the two-flux model can be successfully applied by considering either the total flux transfers (denoted as $S C I$ ) or the Collimated-to-Collimated flux transfers (denoted as $C C$ ). In order to characterize only the diffuse color of a material without direct reflection or transmission, specular excluded measurements (denoted as $S C E$ ) are preferable. However, in the case of translucent layers, the prediction of $S C E$ reflectances and transmittances is not directly possible by using the two-flux model. We show how the two-flux model should be adapted to allow $S C E$ predictions.

After presenting the experimental device and the notations in Section 2, a solution to simulate SCE transfers derived from the twoflux model is established in Section 3 and discussed in Section 4.

\section{Experimental setup and notations}

In our experiments, we used the Color i7 spectrophotometer from X-rite, equipped with a pulse discharge Xenon lamp. Measurements are done from 400 to $750 \mathrm{~nm}$ in steps of $10 \mathrm{~nm}$. For a given sample, the instrument equipped with an integrating sphere supports four measurement geometries, shown in Fig. 1:

- specular included reflectance $\left(\mathrm{di}: 8^{\circ}\right)$, denoted as $r_{S C I}$;

- specular excluded reflectance (de: $\left.8^{\circ}\right)$, denoted as $r_{S C E}$, for which the gloss trap is open;

- total transmittance $\left(\mathrm{d}: 0^{\circ}\right)$, denoted as $t_{S C I}$, for which the sample is placed on the sphere surface;

- collimated-to-collimated regular transmittance $\left(0^{\circ}: 0^{\circ}\right)$, denoted as $t_{C C}$, for which the sample is placed close to the detector.

From the previous measurements, we can also deduce:

- the collimated-to-collimated (specular) reflectance $\left(8^{\circ}: 8^{\circ}\right)$, denoted as $r_{C C}$ :

$$
r_{C C}=r_{S C I}-r_{S C E}
$$

- the transmittance with regular transmittance excluded (de: $\left.0^{\circ}\right)$, denoted as $t_{S C E}$ :

$$
t_{S C E}=t_{S C I}-t_{C C}
$$

Although the light capturing sensor is positioned at $8^{\circ}$ for reflectance measurements and at $0^{\circ}$ for transmittance measurements, we assume hereafter that this small angular shift has no influence.
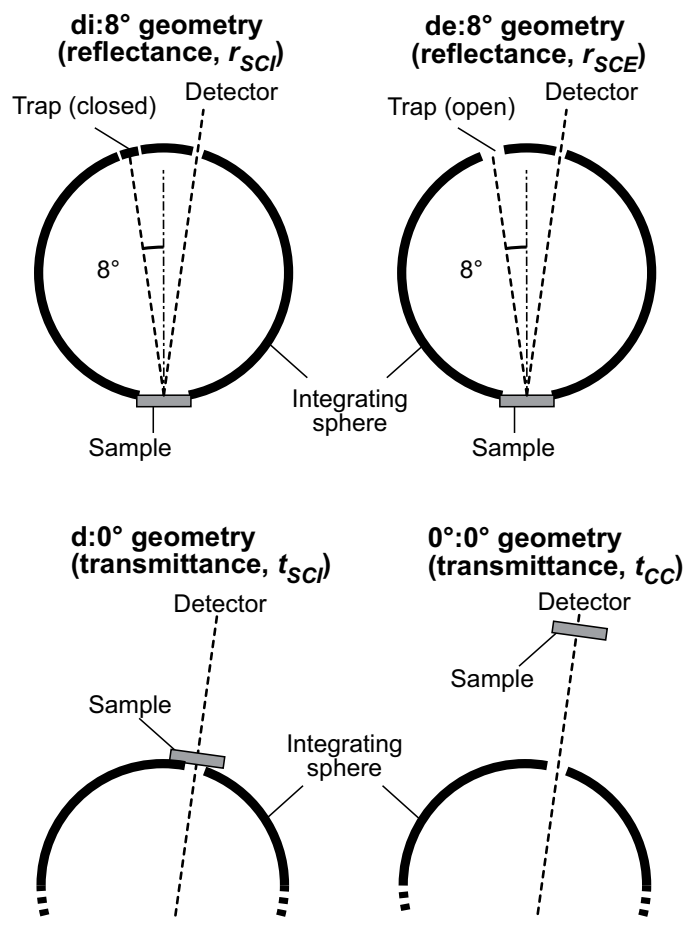

Figure 1. Presentation of the four measurement geometries supported by the integrating sphere spectrophotometer. 
In our experiments, we considered translucent, slightly scattering plastic sheets of red and green color. As shown in Fig. 2, they are placed on top of a white background on which black text is printed in order to put their translucency into evidence.

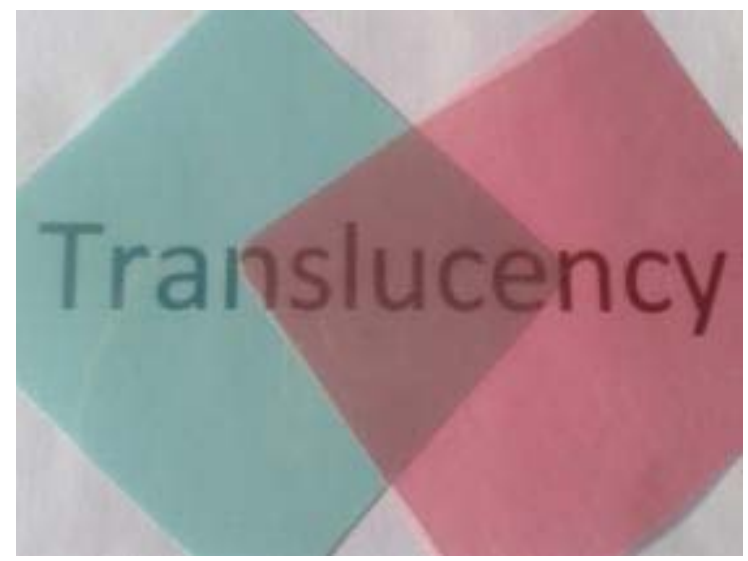

Figure 2. Presentation of the green and red translucent sheets over a white print paper with "Translucency" printed in black.

The measured spectral reflectances and transmittances $r_{S C I}$, $r_{S C E}, t_{S C I}$ and $t_{C C}$ are presented in Figure 3a for one green sheet, and in Figure $3 \mathbf{b}$ for one red sheet. They are the four input spectra for the prediction model. The aim is to predict the reflection and transmission properties of several stacked sheets. Even though the sheets are in mechanical contact with each other, they are not in optical contact: a thin air layer remains between them, and multiple light reflections occur between the sheets. An optical contact obtained by introducing a liquid between the sheets would considerably modify their reflection and transmission properties, therefore their color, in a similar way as shown in Ref. [15] for stacks of nonscattering acetate sheets.

\section{Prediction model}

A two-flux model considers a parallel planar structure of material and therefore reduces the radiative transfer equation to a problem with one spatial dimension. It can be presented as a special case of the $N$-flux model [6] where the radiation field for each position in the stack of sheets is composed of two beams $(N=2)$ propagating forwards $I$ and backwards $J$. Each sheet gives rise to flux transfers characterized by a front side reflectance $r$, a back side reflectance $r$ ', a forward transmittance $t$ and a backward transmittance $t$ '.

Fig. 4 represents the flux transfers for a stack of two sheets (labelled 1 and 2) without optical contact between them, in the framework of the two-flux model.

The resulting reflectances and transmittances for the superposition of the two sheets are (cf. Appendix and [16]):

$$
\left\{\begin{array} { l } 
{ r = r _ { 1 } + \frac { r _ { 2 } t _ { 1 } t _ { 1 } ^ { \prime } } { 1 - r _ { 1 } ^ { \prime } r _ { 2 } } } \\
{ r ^ { \prime } = r _ { 2 } + \frac { r _ { 1 } t _ { 2 } t _ { 2 } ^ { \prime } } { 1 - r _ { 1 } ^ { \prime } r _ { 2 } } }
\end{array} \text { and } \left\{\begin{array}{l}
t=\frac{t_{1} t_{2}}{1-r_{1}^{\prime} r_{2}} \\
t^{\prime}=\frac{t_{1}^{\prime} t_{2}^{\prime}}{1-r_{1}^{\prime} r_{2}}
\end{array}\right.\right. \text {. }
$$

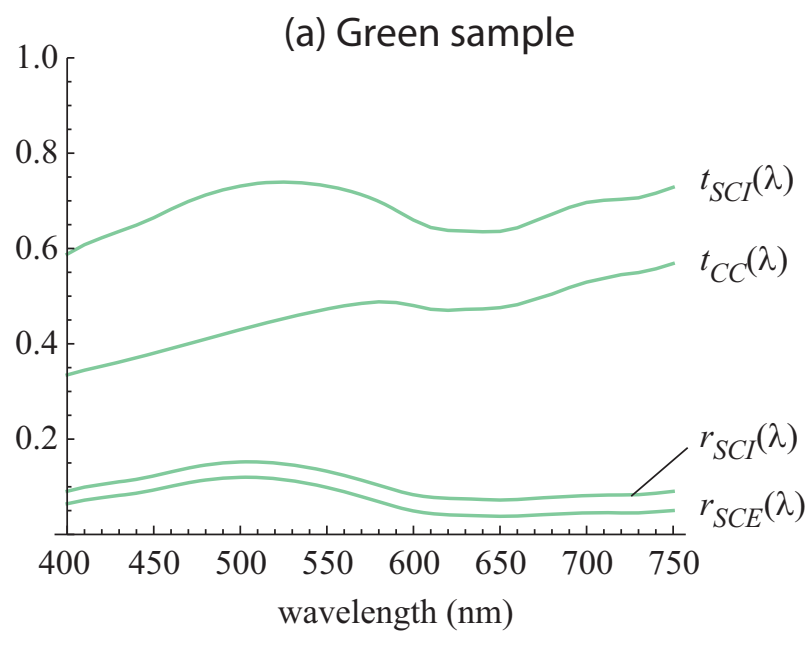

(b) Red Sample

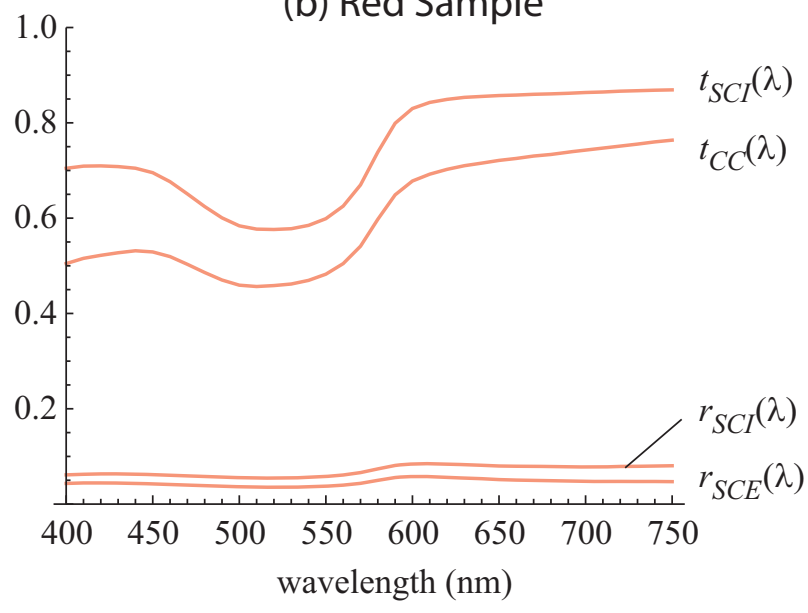

Figure 3. Spectral measurements $r_{S C I}, r_{S C E}, t_{S C I}$ and $t_{C C}$ for a green (a) or a red (b) translucent plastic sheet.In reflectance mode, the sample to be measured is placed in front of a black trap.

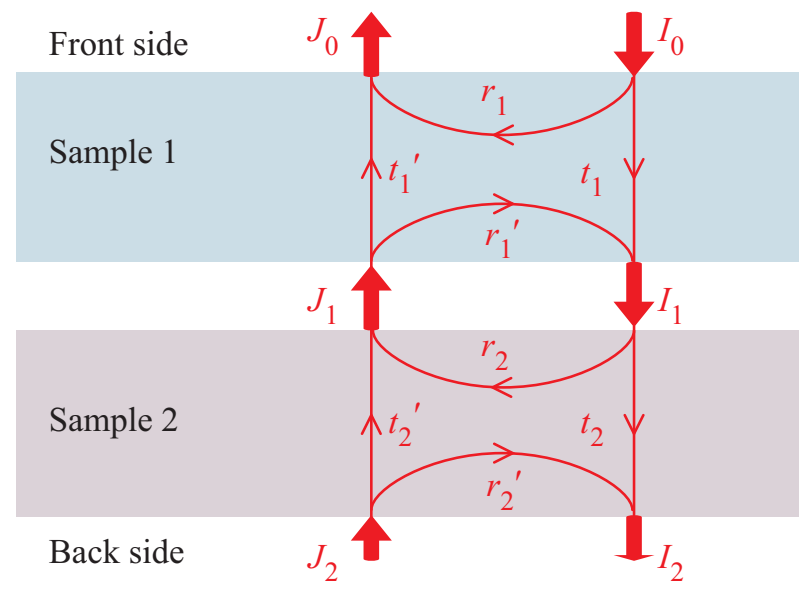

Figure 4. Two-flux transfers for the superposition of two sheets. Bold arrows represent fluxes while thin arrows represent the transfer factors. 
These relations can be generalized to any multilayer system, e.g. by using a matrix formalism [17]. When the sheets are symmetric, i.e. it can be assumed that $r_{1}^{\prime}=r_{1}, t_{1}^{\prime}=t_{1}, r_{2}^{\prime}=r_{2}$, and $t_{2}^{\prime}=t_{2}$, relations (3) simplify as:

$$
\left\{\begin{array}{l}
r=r_{1}+\frac{r_{2} t_{1}^{2}}{1-r_{1} r_{2}} \\
r^{\prime}=r_{2}+\frac{r_{1} t_{2}^{2}}{1-r_{1} r_{2}} \\
t=t^{\prime}=\frac{t_{1} t_{2}}{1-r_{1} r_{2}}
\end{array}\right.
$$

Let us now consider identical symmetric sheets of reflectance $r_{1}$ and transmittance $t_{1}$. By increasing the number of stacked sheets, the resulting reflectance increases and reaches a limit value, denoted as $r_{\infty}$ (cf. Appendix):

$$
r_{\infty}=\alpha-\sqrt{\alpha^{2}-1}
$$

with

$$
\alpha=\frac{1+r_{1}^{2}-t_{1}^{2}}{2 r_{1}} .
$$

This reflectance expression for an infinitely thick material (homogeneous or stratified) is well known and characteristic of the two-flux model. It is also present in the Kubelka-Munk model [2] where $\alpha$ is usually denoted as $a$ and enables computing the albedo of an infinitely thick layer of the material.

It has been shown in Ref. [18] that the two-flux model, and therefore the compositional relations (3), (4) or (5), apply in the two specific $C C$ and $S C I$ configurations, for perfectly transparent media and for strongly diffusing media, respectively.

For translucent layers, the spectral predictions are explained hereafter for the $C C, S C I$ and SCE configurations. The plots of Figure 5 present the measured and predicted reflectances and transmittances of stacks of 2 and 3 identical green sheets and 2 and 3 identical red sheets. The predicted reflectances of semi-infinite stacks of these sheets are also compared with the measurements performed on a stack of 36 green sheets, and on a stack of 52 red sheets. These numbers are chosen in order to reach the stack opacity (the red sheets are more transparent than the green one's). The deviations between the measured and predicted spectra are given in Table 1, and assessed by both the root mean square deviation RMSD and the visual metric CIELAB $\triangle \mathrm{E}_{94}$, obtained by converting both spectra into CIE-1931 XYZ tristimulus values then into CIELAB color coordinates, using the CIE standard illuminant D65.

\section{CC configuration}

In the $C C$ configuration, the incident flux is collimated and only the collimated reflected flux in the specular direction or the collimated transmitted flux in the regular direction is considered. The corresponding reflectances and transmittances are the ones denoted by subscript $C C$. Relations (4) are written:

$$
\left\{\begin{array}{l}
r_{C C}=r_{C C 1}+\frac{r_{C C 2} t_{C C 1}^{2}}{1-r_{C C 1} r_{C C 2}} \\
r_{C C}^{\prime}=r_{C C 2}+\frac{r_{C C 1} t_{C C 2}^{2}}{1-r_{C C 1} r_{C C 2}} \\
t_{C C}=t_{C C}^{\prime}=\frac{t_{C C 1} t_{C C 2}}{1-r_{C C 1} r_{C C 2}}
\end{array} .\right.
$$

Moreover, the relation (5) with $\alpha=\left(1+r_{C C 1}^{2}-t_{C C 1}^{2}\right) / 2 r_{C C 1}$ can be used to compute the $C C$-reflectance for the infinitely thick stack, $r_{C C \infty}$.

The measured and predicted $C C$-reflectances and $C C$ transmittances are given in the first column of the Figure 5. The specular reflectances $r_{C C}$ are almost independent of the number of sheets. They are mainly due to the first reflection at the upper interface of the stack. This is the reason why the spectra are quite achromatic with a low value similar to the $4 \%$ reflectance at normal incidence of an interface between air and a plastic material with refractive index $n=1.5$. The very slight coloration in specular reflection is due to multiple reflections within the first sheet and between the sheets. Given the low values of $r_{C C}$ compared with the other measurements, the predictions are correct for the order of magnitude (RMSD) but not satisfactory in terms of color difference (CIELAB $\Delta$ E94), see Table 1 . This may be explained by the important variability in the surface roughness of the sheets. Moreover, slight measurement errors may be emphasized because the reflectances $r_{C C}$ are not directly measured but deduced from $r_{S C I}$ and $r_{S C E}$ measurements [Eq. (1)].

However, a fairly good agreement is observed between measured and predicted spectral $C C$-transmittances for 2 and 3 sheets. The $C C$-transmittances are induced by light absorption by the material as well as light scattering by either the material or its interfaces with air. Light scattering has the effect of spreading initially collimated light into a larger solid angle. In the case of two or more sheets, the attenuations due to absorbance and solid angle spreading are multiplicative, especially when the denominator $1-r_{C C 1} r_{C C 2}$ in Eq. (6) is close to 1, i.e. when the single-sheet reflectances are low. This allows understanding why the predictions for $C C$-transmittances are accurate.

Compared to the specular reflectances, the regular transmittances are more colorful, but decrease rapidly towards zero as the number of sheets increases."

\section{SCI configuration}

In the SCI configuration, the incident flux is perfectly diffuse and the light capturing sensor is at the sheet normal. This mode provides the SCI-reflectances and -transmittances that we assume to correspond to the total reflected or transmitted fluxes exiting from the sample. The two-flux model also applies. For the specular included transfer factors, denoted by subscript $S C I$, the relations (4) become:

$$
\left\{\begin{array}{l}
r_{S C I}=r_{S C I 1}+\frac{r_{S C I 2} t_{S C I 1}^{2}}{1-r_{S C I 1} r_{S C I 2}} \\
r_{S C I}^{\prime}=r_{S C I 2}+\frac{r_{S C I 1} t_{S C I 2}^{2}}{1-r_{S C I 1} r_{S C I 2}} \\
t_{S C I}=t_{S C I}^{\prime}=\frac{t_{S C I 1} t_{S C I 2}}{1-r_{S C I 1} r_{S C I 2}}
\end{array}\right.
$$




\section{(a) Green samples}
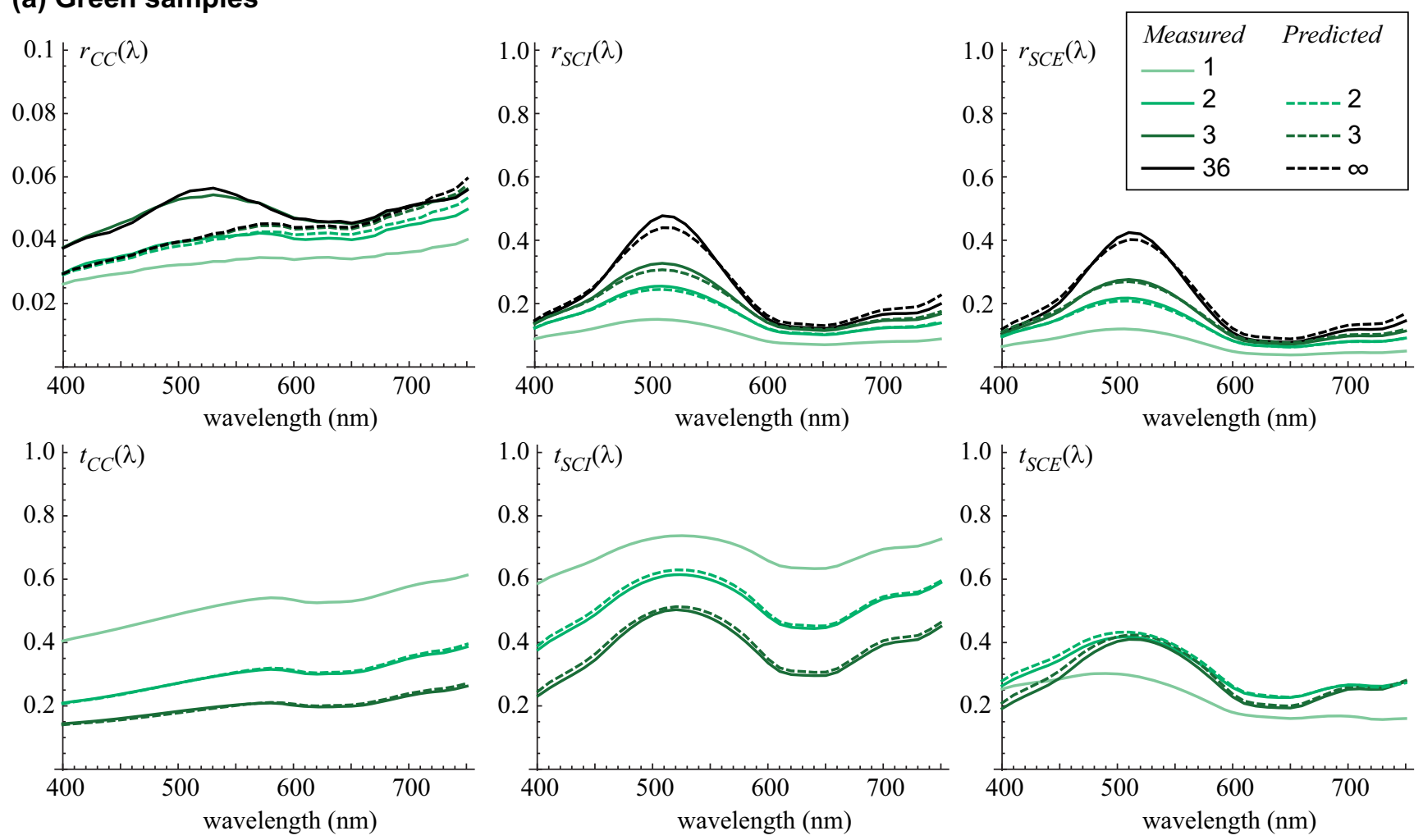

\section{(b) Red samples}
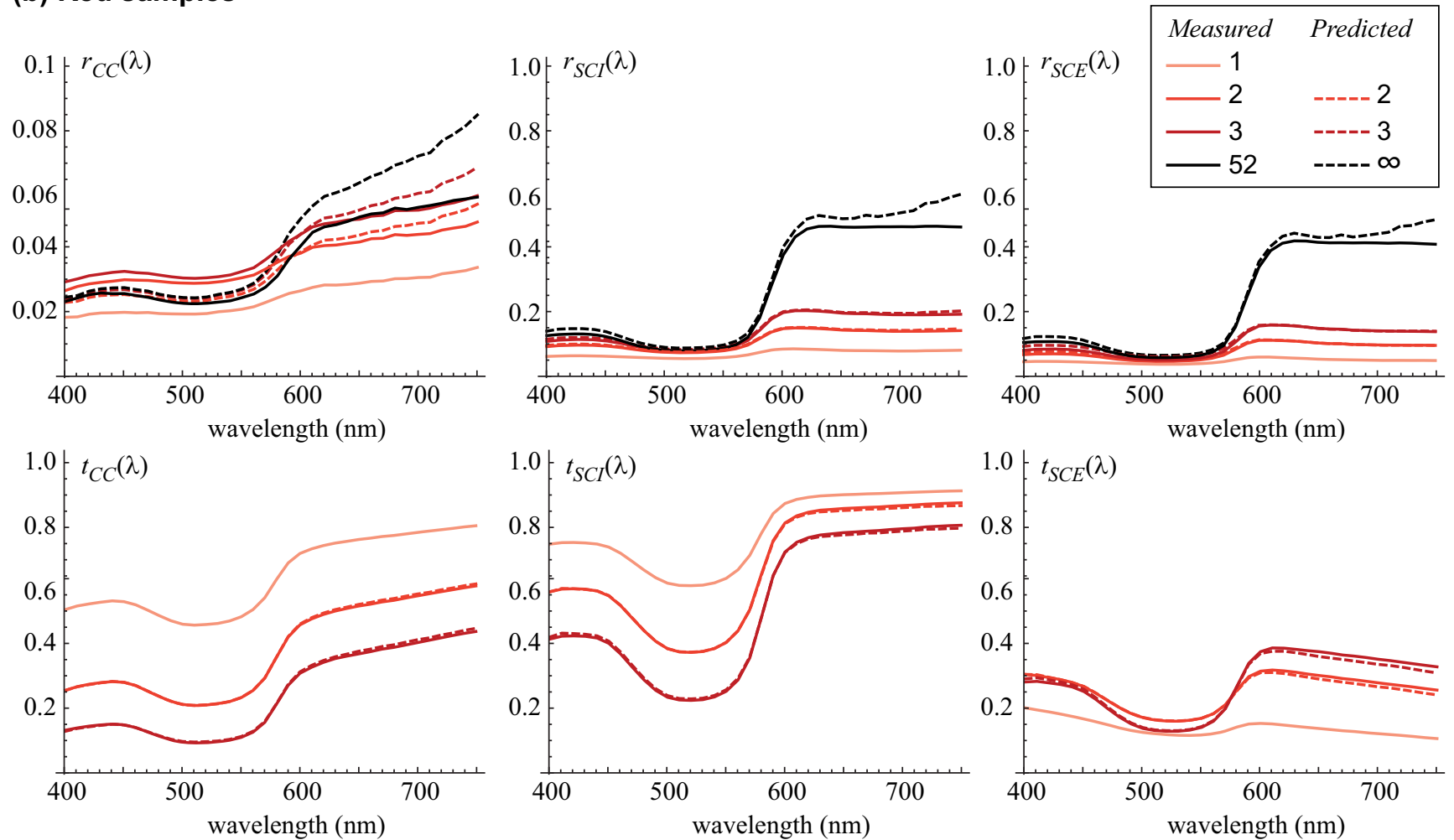

Figure 5. Measured and predicted spectral reflectances and transmittances of stacks of green or red sheets in Collimated-to-Collimated (left column), Specular Component Included (middle column) and Specular Component Excluded (right column) configurations. The numbers indicate the number of stacked sheets. 
Table 1. RMS deviations between the measured and predicted spectra and equivalent color deviations $\left(\Delta \mathrm{E}_{94}\right)$.

\begin{tabular}{|c|c|c|c|c|c|c|c|c|c|c|c|c|c|}
\hline & & \multicolumn{4}{|c|}{ Collimated-to-Collimated } & \multicolumn{4}{|c|}{ Specular Component Included } & \multicolumn{4}{|c|}{ Specular Component Excluded } \\
\hline & & \multicolumn{2}{|c|}{$r_{C C}$} & \multicolumn{2}{|c|}{$t_{C C}$} & \multicolumn{2}{|c|}{$r_{S C I}$} & \multicolumn{2}{|c|}{$t_{S C I}$} & \multicolumn{2}{|c|}{$r_{S C E}$} & \multicolumn{2}{|c|}{$t_{S C E}$} \\
\hline & & RMSD & $\Delta \mathrm{E}_{94}$ & RMSD & $\Delta \mathrm{E}_{94}$ & RMSD & $\Delta \mathrm{E}_{94}$ & RMSD & $\Delta \mathrm{E}_{94}$ & RMSD & $\Delta \mathrm{E}_{94}$ & RMSD & $\Delta \mathrm{E}_{94}$ \\
\hline \multirow{3}{*}{ 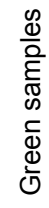 } & 2 & 0.0016 & 0.97 & 0.0049 & 0.63 & 0.0045 & 0.95 & 0.0123 & 0.83 & 0.0039 & 0.99 & 0.0113 & 1.05 \\
\hline & 3 & 0.0080 & 4.26 & 0.0053 & 1.64 & 0.0090 & 1.54 & 0.0139 & 1.03 & 0.0056 & 1.25 & 0.0142 & 1.41 \\
\hline & 36 & 0.0081 & 4.68 & - & - & 0.0164 & 1.94 & - & - & 0.0143 & 1.84 & - & - \\
\hline \multirow{3}{*}{$\begin{array}{l}\mathscr{d} \\
\frac{0}{0} \\
\tilde{E} \\
\mathbb{D} \\
\infty \\
\mathbb{d} \\
\mathscr{d}\end{array}$} & 2 & 0.0040 & 2.86 & 0.0034 & 0.15 & 0.0029 & 0.44 & 0.0042 & 0.15 & 0.0053 & 1.89 & 0.0076 & 0.56 \\
\hline & 3 & 0.0051 & 2.99 & 0.0053 & 0.54 & 0.0051 & 0.77 & 0.0061 & 0.42 & 0.0078 & 2.22 & 0.0103 & 0.87 \\
\hline & 52 & 0.0151 & 2.41 & - & - & 0.0356 & 1.76 & - & - & 0.0256 & 1.59 & - & - \\
\hline
\end{tabular}

The SCI-reflectance of an infinite stack of sheets, $r_{S C I \infty}$, is also given by relation (5) with $\alpha=\left(1+r_{S C I 1}^{2}-t_{S C I 1}^{2}\right) / 2 r_{S C I 1}$.

The second column of Figure 5 presents the measured and predicted SCI-reflectances and -transmittances of stacks of identical sheets. As the number of sheets in the stack increases, the diffuse reflectance monotonously increases, and the diffuse transmittance monotonously decreases. The prediction accuracy, presented in Table 1 (central columns), is fairly good except in the case of an infinitely thick stack for which we denote a sensible deviation in the spectral domain where the stack is the more reflecting. Remind that the reflectance of the infinitely thick stack of sheets, $r_{S C I \infty}$, is predicted from the measured reflectance $r_{S C I 1}$ and transmittance $t_{S C I 1}$ of one sheet. A slight error in the spectral measurements may induce a significant deviation in the predicted reflectance $r_{S C I_{\infty}}$. We often observe that the prediction accuracy for $r_{S C I \infty}$ is improved by using the measured reflectance and transmittance of $k=2$ or more stacked sheets and the following equation (cf. Eq. (14) of the Appendix)

$$
r_{\infty}=r_{k}+\frac{t_{k}^{2} r_{\infty}}{1-r_{k} r_{\infty}} .
$$

In contrast with the $C C$-transfer factors, $S C I$-reflectances are not negligible and SCI-transmittances of two or more samples [Eqs. (7)] cannot be reduced to a multiplicative law. The good predictions given by Table 1 require taking into account the multiple reflections between the sheets.

\section{SCE configuration}

In the $S C E$ configuration, we consider only the scattered out from the regular directions. In contrast with the $C C$ configuration where only collimated fluxes are considered, and the $S C I$ configuration where total fluxes are considered, we experimentally observed that the two-flux model does not apply with the $S C E$ flux transfers. However, thanks to relations (1) and (2), the $S C E$ transfers can be deduced from the $C C$ transfers, given by Eqs. (6), and the $S C I$ transfers, given by Eqs. (7). We have:

$$
\left\{\begin{array}{l}
r_{S C E}=r_{S C I}-r_{C C} \\
r_{S C E}^{\prime}=r_{S C I}^{\prime}-r_{C C}^{\prime} \\
t_{S C E}=t_{S C E}^{\prime}=t_{S C I}-t_{C C}
\end{array} .\right.
$$

Similarly, the infinitely thick $S C E$-reflectance $r_{S C E \infty}$ is deduced as:

$$
r_{S C E \infty}=r_{S C I \infty}-r_{C C \infty} \text {. }
$$

It is worth noting that the $S C E$ measurements of the individual sheets are not directly required for the prediction of the $S C E$ reflectances and transmittances of a stack [Eqs. (8) and (9)]. However, to obtain the $r_{C C}$ reflectances [Eq. (1)], the $r_{S C E}$ reflectances of the individual sheets are to be measured.

The right column in Figure 5 shows the measured and predicted $S C E$-reflectances and -transmittances for stacks of identical sheets. The predictions are obtained by first predicting the $C C$ and $S C I$ transfer factors of multiple sheets and then obtaining the SCE transfer factors according to Eqs. (8) and (9). The predictions are as good as for the $S C I$ configuration (Table 1).

In contrast with $C C$ and $S C I$ measurements, for a given wavelength, the $S C E$ transfers do not always vary monotonously as the number of sheets increases. It is particularly noticeable for the transmittances $t_{S C E}$ in Figure 5. As the two-flux model is a radiative flux balance, it can only predict monotonous variations of the flux transfer factors according to the number of sheets. Due to the observed non-monotonic property, one cannot directly use the $S C E$ measurements to predict with the two-flux model the $S C E$ reflectances and transmittances. However, by first predicting $S C I$ and $C C$ reflectances and transmittances according to Eqs. (6-7), we obtain with Eq. (8) valid SCE reflectance and transmittance predictions. 
Therefore, the observed non-monotonic property is an indication that the two-flux model cannot directly apply on SCE measurements with this kind of material.

Figure 6 shows the $S C E$-reflectance and -transmittance of the red translucent plastic sheet on top of the green translucent plastic sheet, and the ones of the green sheet on top of the red sheet. The measured spectra are compared with the spectra predicted by using equations (6), (7) and (8), from the measurements made on one sheet and plotted in Figure 3. As expected, the transmittances are independent of the stacking order, while the resulting reflectance looks reddish or greenish according to whether the red or respectively the green sheet is closer to the observer. Table 2 presents the spectral RMS deviations and the equivalent color deviations (CIELAB $\triangle \mathrm{E}_{94}$ ) between the measured and predicted spectra. It also shows the totally wrong predictions that would be obtained by applying the two-flux model [Eq. (4)] directly with the $S C E$-reflectance and -transmittance of the individual samples.
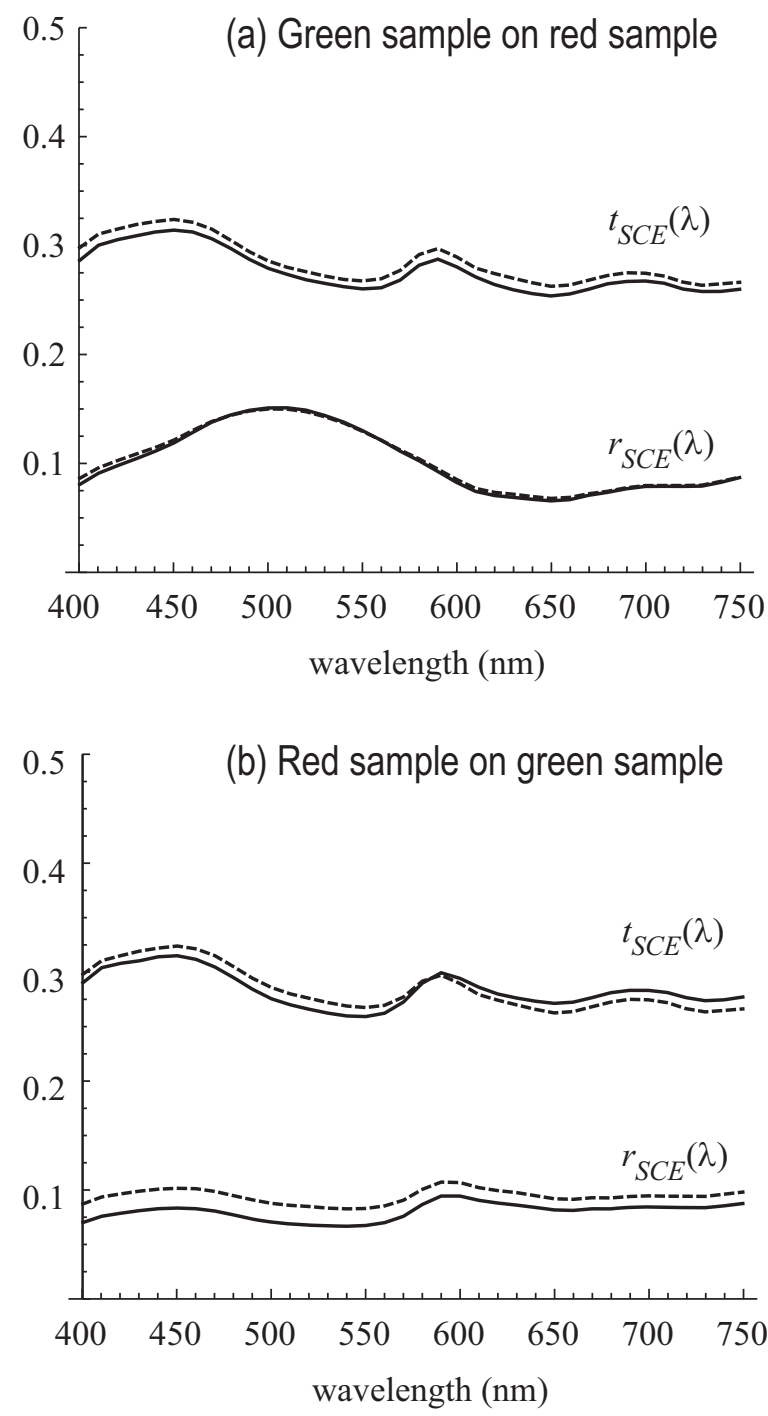

Figure 6. Measured (solid lines) and predicted (dotted lines) spectral SCEreflectances and -transmittances of one green translucent sheet on top of one red translucent sheet.
Table 2. RMS deviations between the measured and predicted spectra and equivalent color deviations $\left(\Delta \mathrm{E}_{94}\right)$ for the SCE configuration.

\begin{tabular}{ccccc}
\hline \hline & \multicolumn{2}{c}{$r_{S C E}$} & \multicolumn{2}{c}{$t_{S C E}$} \\
\hline & $\mathrm{RMSD}$ & $\Delta \mathrm{E}_{94}$ & $\mathrm{RMSD}$ & $\Delta \mathrm{E}_{94}$ \\
\hline $\begin{array}{c}\text { Green sheet on top of } \\
\text { the red sheet }\end{array}$ & 0.0023 & 0.82 & 0.0085 & 0.79 \\
\cline { 2 - 5 } & $0.0271^{*}$ & $5.36^{*}$ & $0.229^{*}$ & $33.4^{*}$ \\
\hline $\begin{array}{c}\text { Red sheet on top of the } \\
\text { green sheet }\end{array}$ & 0.0144 & 3.33 & 0.0084 & 1.55 \\
\cline { 2 - 5 } & $0.0325^{*}$ & $7.91^{*}$ & $0.237^{*}$ & $33.9^{*}$ \\
\hline \hline
\end{tabular}

* predictions performed by using directly Eq. (4) with the measured SCE spectral reflectances and transmittances of the individual sheets.

\section{Discussion}

In this section, we propose to discuss the applicability of the extended two-flux model, in particular in the extreme cases of almost non-scattering and strongly scattering materials. We then address the limitations of the proposed method, firstly due to the use of single-integrating-sphere measurements, secondly due to the difficulty induced by the progressive spreading of a collimated beam.

\subsection{Special cases}

We can verify that the model presented in the previous sections reduces to the same two-flux model in the special cases of nonscattering or strongly scattering materials. In absence of scattering in the material (transparent layers with flat interfaces), either the incident light is collimated and only $C C$ transfers occur [Eqs. (6) applies], or the incident light is diffuse and total $S C I$ transfers must be considered [Eqs. (7)]. For strongly scattering samples, the same reflectances and transmittances are measured whether the incident light is collimated or diffuse. Therefore, there are no $C C$ transfers, $S C I$ transfers are equivalent to $S C E$ transfers and given by Eqs. (7). This is however a simplified view. If for example the scattering sheets have flat interfaces, the $S C I$ - and $S C E$-reflectances differ by a small value, almost the same at all wavelengths, corresponding to the Fresnel reflectance of the front sheet surface. In order to take this phenomenon into account, Equations (8) can be modified as follows:

$$
\left\{\begin{array}{l}
r_{S C E}=r_{S C I}-r_{C C 1} \\
r_{S C E}^{\prime}=r_{S C I}^{\prime}-r_{C C 2} \\
t_{S C E}=t_{S C E}^{\prime}=t_{S I}
\end{array}\right.
$$

where the Fresnel reflectance of the front surface, $r_{C C 1}$ or $r_{C C 2}$ , depends on the surface roughness [19]. The values for $r_{C C 1}$ or $r_{C C 2}$ decrease as the surface roughness increases, and the difference between the $S C I$ and $S C E$ reflectances thus decreases towards zero, the limit case where the light reflected by a sheet having a rough interface can be considered as Lambertian.

Our experiments were carried out with stacks of identical translucent sheets without optical contact, but similar experiments could be performed by stacking translucent layers in optical contact [15]. The same model applies but with the material considered without its bordering interfaces with air. The optical effect of the bordering interfaces, called Saunderson's correction in the two-flux approach [20], depends on the measurement geometry as expressed in [17]. 


\subsection{Single-integrating-sphere limitation}

According to the known limitations of the two-flux theory, we would expect that Eqs. (7) apply for the diffuse-to-diffuse transfers ( $D D$-reflectances and -transmittances, for which the incident light is diffuse, and the outgoing light is captured over the hemisphere), but strictly not for the $S C I$ transfers. Rigorous measurements of the $D D$ transfers would require a double-integrating-sphere [21]. Moreover, a double-integrating-sphere would ideally enable three independent measurements, for both reflectance and transmittance: collimatedto-collimated, diffuse-to-diffuse, as well as collimated-to-diffuse configurations. These six independent measurements would allow calibrating a four-flux model, which consists in adding two collimated fluxes to the two diffuse fluxes [6-10]. It can be shown [11] that the four-flux model is equivalent to the two-flux model for both $C C$ and $D D$ transfers but not for the collimated-to-diffuse transfers. Unfortunately, double-integrating-sphere measurements are very rarely proposed by current commercial spectrometric devices. With a much more common single-integrating-sphere allowing four independent measurements (Figure 1), the adapted two-flux method presented in this paper seems to be appropriate for predicting the $C C, S C I$ and $S C E$ transmittances and reflectances of superposed slightly diffusing flat sheets.

\subsection{Progressive "decollimation"}

The samples presented in this paper have almost flat interfaces: their surface scattering can be neglected without loss of accuracy. When crossing a sample with flat interfaces, collimated light is either not scattered and stays collimated, or is scattered within the sheets' bulk. A more detailed analysis, based on the samples BRDF's and BTDF's may help understanding in depth how light is scattered and why the predictions are accurate in $C C, S C I$ and $S C E$ modes. With samples presenting rough interfaces, the surface scattering makes a collimated incident light beam spread over a larger solid angle, which grows as the number of encountered interfaces increases. Neither the two- nor the four-flux model can render this angular spreading of the beam, especially if measurements based on the integrating sphere (or even two) are used. It is hard to predict which part of the initially collimated flux can be considered as outside of the specular port of the sphere, therefore as part of the diffuse flux. This is especially difficult because the gloss trap dimensions are not standardized. However, modeling, as simply as possibly (i.e. without use of a complete bidirectional description of light scattering by the sample), this "decollimation" of initially collimated light while crossing a translucent material is an interesting topic for future research.

\section{Conclusion}

The aim of this study was to show that the diffuse color of translucent materials can be characterized by a simple extension of a two-flux model and by measurements made with common affordable instruments whose geometry is conceived for strongly scattering media. The proposed extension of the two-flux model to diffuse specular excluded reflectances and transmittances replaces more complex scattering theories and goniospectrophotometers which require much expertise to be used in practical applications. The proposed method relies on measurements performed by using an integrating sphere in specular included and excluded modes. The prediction of the reflectances and transmittances of stacks of samples knowing those of each individual sample can be done by applying the two-flux model for, on one hand, the specularcomponent-included reflectance and the regular-component- included transmittance (SCI transfers), and on the other hand, for the collimated-to-collimated reflectance and transmittance $(C C$ transfers). The specular-component-excluded reflectance and regular-component-excluded transmittance ( $S C E$ transfers) can then be deduced by subtracting the $S C I$ and $C C$ transfers, either measured or predicted by the extended two-flux model. This simple method enables distinguishing the color variations according to the material thickness both in the regular directions ( $C C$ transfers) and in the rest of the hemisphere (SCE transfers). From the experiments we carried out with slightly scattering polymer sheets, we observed that the simulations are in good agreement with the spectral measurements and allow good predictions of the specular reflected and regular transmitted colors as well as of the reflected and transmitted diffuse colors (in specular excluded mode). This model could be used for innovative printing systems like some 3D printers, for which the color synthesis is based on translucency. This radiometric study also offers new tools for analyzing the color and the perception of translucent samples.

\section{APPENDIX: The two-flux model}

Fig. 4 represents the flux transfers for a stack of two samples in the framework of the two-flux model. Let us consider the sample labelled 1. The fluxes $I_{0}$ and $J_{1}$ illuminating it, and the outgoing fluxes $J_{0}$ and $I_{1}$ can be related according to the following equations:

$$
\left\{\begin{array}{l}
J_{0}=r_{1} I_{0}+t_{1}^{\prime} J_{1} \\
I_{1}=t_{1} I_{0}+r_{1}^{\prime} J_{1}
\end{array} .\right.
$$

Similar relations can be written for the sample labelled 2 between the fluxes $I_{1}, J_{2}$ and $J_{1}, I_{2}$ :

$$
\left\{\begin{array}{l}
J_{1}=r_{2} I_{1}+t_{2}^{\prime} J_{2} \\
I_{2}=t_{2} I_{1}+r_{2}^{\prime} J_{2}
\end{array} .\right.
$$

The flux transfers are obtained by combining the relations (11) and (12) :

$$
\left\{\begin{array}{l}
r=\frac{J_{0}}{I_{0}}=r_{1}+\frac{r_{2} t_{1} t_{1}^{\prime}}{1-r_{1}^{\prime} r_{2}} \\
r^{\prime}=\frac{I_{2}}{J_{2}}=r_{2}+\frac{r_{1} t_{2} t_{2}^{\prime}}{1-r_{1}^{\prime} r_{2}} \\
t=\frac{I_{2}}{I_{0}}=\frac{t_{1} t_{2}}{1-r_{1}^{\prime} r_{2}} \\
t^{\prime}=\frac{J_{0}}{J_{2}}=\frac{t_{1}^{\prime} t_{2}^{\prime}}{1-r_{1}^{\prime} r_{2}}
\end{array}\right.
$$

As an alternative to the radiative flux balance, equations (13) can be obtained by writing the multiple reflections between the two samples as a geometric series [4].

Let us now consider a semi-infinite stack of identical symmetric slices, e.g. translucent sheets stacked together. Each slice has a reflectance $r_{1}=r_{1}^{\prime}$ and transmittance $t_{1}=t_{1}^{\prime}$, independent of the measured side. When many slices are stacked with each other, the stack reflectance reaches an invariant value, denoted as $r_{\infty}$, which satisfies the following relation issued from (13) by 
considering that adding one slice does not modifies this reflectance value:

$$
r_{\infty}=r_{1}+\frac{t_{1}^{2} r_{\infty}}{1-r_{1} r_{\infty}} .
$$

This relation may also be written:

$$
r_{\infty}^{2}-2 \alpha r_{\infty}+1=0
$$

with

$$
\alpha=\frac{1+r_{1}^{2}-t_{1}^{2}}{2 r_{1}} \text {. }
$$

Eq. (15) has one solution lower than 1, therefore valid for a reflectance:

$$
r_{\infty}=\alpha-\sqrt{\alpha^{2}-1}
$$

\section{Aknowledgement}

This work was supported by the French National Research Agency (ANR) in the framework of project PHOTOFLEX $n^{\circ}$ ANR12-NANO-0006.

\section{References}

[1] G.G. Stokes, On the intensity of the light reflected from or transmitted through a pile of plates, Proc. of the Royal Society of London 11, 545-556 (1860).

[2] P. Kubelka and F. Munk, Ein Beitrag zur Optik der Farbanstriche, Zeitschrift für technische Physik 12, 593-601 (1931).

[3] P. Kubelka, New contributions to the optics of intensely lightscattering material, part I, J. Opt. Soc. Am. 38, 448-457 (1948).

[4] P. Kubelka, New contributions to the optics of intensely lightscattering material, part II. Non-homogeneous layers, J. Opt. Soc. Am. 44, 330-334 (1954).

[5] S. Chandrasekhar, Radiative transfert, Dover, New-York (1960).

[6] P.S. Mudgett and L.W. Richards, Multiple scattering calculations for technology, Appl. Opt. 10, 1485-1502 (1971).

[7] J.K. Beasley, J.T. Atkins and F.W. Billmeyer, Scattering and absorption in turbid media, in Electromagnetic scattering Eds R.L. Rowell and R.S. Stein, Gordon and Breach, New York, 765-785 (1967)

[8] A. Ishimaru, Wave Propagation and Scattering in Random Media, Academic Press, New York (1978).

[9] B. Maheu, J.N. Le Toulouzan and G. Gouesbet, Four-flux models to solve the scattering transfer equation in terms of Lorenz-Mie parameters, Appl. Opt. 23, 3353-3362 (1984).

[10] B. Maheu and G. Gouesbet, Four-flux models to solve the scattering transfer equation. Special cases, Appl. Opt. 25, 1122-1228 (1986).
[11] L. Simonot, R.D. Hersch, M. Hébert, S. Mazauric, Multilayer fourflux matrix model accounting for directional-diffuse light transfers, Appl. Opt. 55, 27-37 (2016).

[12] CIE, Colorimetry, 3rd ed., Technical Report CIE 15 (CIE, 2004).

[13] R. Ulbricht, Die Bestimmung der mittleren räumlichen Lichtintensität durch nur eine Messung, Elektrotech. Z. 21, 595-597 (1900).

[14] CIE, Geometric tolerances for colour measurements, Technical Report CIE 176 (2006).

[15] M. Hébert, R.D. Hersch, L. Simonot, Spectral prediction model for piles of nonscattering sheets, J. Opt. Soc. Am. A 25, 2066-2077 (2008).

[16] G. Kortüm, Reflectance spectroscopy (principles, methods and applications), Springer-Verlag, New-York (1969).

[17] S. Mazauric, L. Simonot, M. Hébert and T. Fournel, Two-flux transfer matrix model for predicting the reflectance and transmittance of duplex halftone prints, JOSA A 31, 2775-2788 (2014).

[18] M. Hébert, R.D. Hersch, J.-M.Becker, Compositional reflectance and transmittance model for multilayer specimens, J. Opt. Soc. Am. A 24, 2628-2644 (2007).

[19] L. Simonot, M. Elias, Color change due to surface state modification, Col. Res. and appl. 28 45-49 (2003).

[20] J.L. Saunderson, Calculation of the color pigmented plastics, J. Opt. Soc. Am. 32, 727-736 (1942).

[21] J.W. Pickering, C.J.M. Moes, H.J.C.M. Sternborg, S.A. Prahl, J.C. van Gemert, Two integrating spheres with an intervening scattering sample, J. Opt. Soc. Am. A 9, 621-631 (1992).

\section{Author Biography}

Lionel Simonot completed his PhD studies at the Centre de Recherche et de Restauration des Musées de France (C2RMF), located in the Palais du Louvre in Paris, where he studied the light scattering by painting glazes. He obtained the doctor grade in 2002. Since 2003, he is assistant professor at the Institut Pprime (CNRS UPR 3346 and University of Poitiers). His research activities focus on the optical properties of materials at different scales (nanocomposite layers, scattering layers, flat or rough interfaces).

Mathieu Hébert completed his PhD studies at the Ecole Polytechnique Fédérale de Lausanne (EPFL, Switzerland) and obtained the doctor grade in 2006. Since 2010, he is assistant professor at the Institut d'OptiqueGraduate School and at the Laboratoire Hubert Curien of CNRS and University Jean Monnet of Saint-Etienne. His research activity is focused on optical models for predicting the visual rendering of colored surfaces.

Serge Mazauric is professor of mathematics and physics in the engineering school CPE-Lyon. He completed in 2016 his PhD studies at the University of Saint-Etienne, France. His work focuses on physical models for computational printing.

Roger David Hersch is a honorary professor of computer science at the Ecole Polytechnique Fédérale de Lausanne (EPFL), Switzerland. Together with his former PhD students, he developed novel imaging techniques related to color reproduction, color prediction, moiré imaging and visual document security. He is a fellow of IS\&T. 